\title{
Editorial: The Making of PRL: Mission, Material, Method
}

We at Physical Review Letters (PRL) are pleased to celebrate the 125th anniversary of our parent journal, The Physical Review (journals.aps.org/125years). Over the years, that original publication has evolved into the APS Physical Review journal collection, and our relationship with these partner journals continues to be strong. Every year, our journals share the vetting of thousands of manuscripts and work together to improve the review process and the way papers are disseminated.

PRL's origins lie in a section called "Letters to the Editor" that debuted in The Physical Review in 1929. In 1958, responding to the growth of the section, then-Managing Editor Samuel Goudsmit spun those Letters off into a separate journal, Physical Review Letters. The year 2018 thus marks another anniversary, the 60th of PRL. The past 60 years have been very successful and as we begin our next 60 years, we look back at how PRL has evolved and share our vision for the future.

Consider PRL's original mission. Goudsmit conceived of PRL as a way to inform physicists of important developments across all of physics in a short format accessible to a broad readership [1]. It was an immediate success, and the model continues to serve PRL well. Important Letters across all subfields of physics have graced its pages over the past six decades, including those associated with many Nobel Prizes in physics (and a few in chemistry). We thank our authors for their continued submission of excellent results, and our reviewers for their service to us and to the community.

Our mission today is still very much the same: to provide a marketplace for physics research, where readers can find the crucial results across every subfield, not just those that are hot. This requires a careful balance-we must publish many good papers across all of physics, but not so many that individual papers get lost, and not in numbers that become indigestible for readers. For this reason we recently pushed again to raise the bar for publication in PRL, and now publish about 50 Letters per week. For now this is a good number that we aim to maintain.

Beyond its mission, the material PRL publishes has evolved, and is now quite different from what the early issues offered. The original content was more focused on nuclear and particle physics. Condensed matter physics was then a small component, and many topics that are prevalent today, such as quantum information, biological physics, and cold atom physics, did not yet exist. We strive to ensure that the scope of the journal matches the interest of the authors and readers. We are a physics journal, but today's physics research is much more about interwoven disciplines - physics with chemistry, biology, materials science, and others. So, we ensure that PRL is also a home for the best interdisciplinary work that is significant for physics.

Another change in published material is the format. The original idea was that Letters be around 800 words, but this strict length limit was only enforced in 1966. The 4-page limit we use today is similar to that imposed in 1971 [2], even as ink-on-paper has given way to online publication, and we view it as beneficial: short, self-contained articles are often better written, a plus for busy readers.

As for method, our principal tool is peer review. Early on, with no web hosting or arXiv preprint repository, publication speed was more of an issue, and there was little time for detailed peer review. Editors reviewed papers themselves, or obtained minimal input from local colleagues. As submissions grew, editors and their local contacts were overwhelmed, and almost all manuscripts were reviewed externally. More recently, the number of submissions again grew too large to handle, and we now send about three-quarters of submissions out for external review. We carefully select these, often seeking advice from other editors or an Editorial Board member. Other journals that are very selective seek expert input on a much smaller fraction of submissions.

The PRL reviewers have a different role as compared to other journals. Of course we ask them to comment on the validity of the manuscripts and suggest improvements as applicable. In addition, we want them to remark on importance, interest, and relevance for PRL [3]. One constant throughout PRL's history is that editors take the input from reviewers as advice [4]. 
Quite often the recommendations are not unanimous and the final decision is then made after weighing all available input.

Editors of the Physical Review journals have also introduced various highlighting mechanisms for a selection of published articles. At PRL, the editors choose about one Letter in six as an Editors' Suggestion. These Letters are specifically marked on the PRL website and accompanied by a tweet from @ PhysRevLett. Many Letters are also covered by the APS online publication Physics, which publicizes interesting results for a broad audience, including a large network of journalists.

Finally, PRL, like the rest of the Physical Review family and physics itself, has become very international. About $70 \%$ of PRL submissions and published Letters now come from outside the U.S. The composition of our editorial team reflects the broad international distribution of authors. Today the PRL editors hail from twelve countries. We feel that it is important for the editors who handle manuscripts to represent the authors who submit them.

Over the last 60 years, PRL has become the global, go-to physics journal, offering a unique combination of breadth, quality, and long-term value. We expect the globalization of PRL to continue, and to stay connected to this community, many of our editors travel around the world to attend conferences and give talks. The journal is fortunate to have an excellent team of talented and experienced professional editors. If you see one of us, ask questions, give feedback, and pass along your thoughts (or email prl@aps.org). We will be happy to respond. Our success relies on the whole physics community. With continued community support, we foresee a bright new 60-year cycle for PRL.

Hugues Chaté

Reinhardt Schuhmann

Published 7 February 2018

DOI: 10.1103/PhysRevLett.120.060001

[1] S. A. Goudsmit and S. Pasternak, Editorial, Phys. Rev. Lett. 2, 1 (1959).

[2] S. A. Goudsmit and G. L. Trigg, Troubles with Length, Phys. Rev. Lett. 27, 1 (1971).

[3] S. A. Goudsmit, How Wide is Gray?, Phys. Rev. Lett. 31, 669 (1973).

[4] S. A. Goudsmit and G. L. Trigg, Referee Reports, Phys. Rev. Lett. 24, 251 (1970).

PRL Lead Editor Hugues Chaté is a senior scientist at CEA-Saclay, France, and Chair Professor at the Beijing Computational Science Research Center in China. Editor Reinhardt Schuhmann has been with PRL full-time for more than 25 years.

[This information appeared previously in APS News (aps.org/publications/APSnews).] 